\title{
Standards and Guidelines for Personal Health Records in the United States: Finding Consensus in a Rapidly Evolving and Divided Environment
}

Binh Q. Tran* and Pedro Gonzales

Department of Biomedical Engineering, The Catholic University of America, 620 Michigan Ave, NE ,Washington, DC 20064

\begin{abstract}
Health care spending in the U.S. over the past decade has dramatically increased over the past decade and is expected to rise to $20 \%$ of GDP in the United States by the end of the decade. In 2010, the U.S. Congress approved the Health Care Reform Act with a critical component of this legislation being the adoption of health information technologies (i.e. EMRs, EHRs, PHRs, etc.) aimed at transforming the health care system. Consumer-driven health care models are seen as essential to control the escalating costs of health care. The objective of this research is to canvas the landscape of existing standards and guidelines relating to electronic personal health records (PHRs) and to evaluate the level of adoption of these standards and guidelines amongst current vendors. Through this effort, we propose a consensus standard for PHRs consisting of 14 data components, 11 of which should be essential for all PHRs and 3 additional recommended data components. Through a survey of existing PHR vendors, we observe a low level of adoption of existing standards in commercially available PHR products and note a wide variation (36-73\%) of inclusion of critical and desired data components. Further, we propose 4 key features and services based upon a review of the existing literature to facilitate consumer adoption and to improve usefulness of PHRs. By proposing a consensus standard for PHR data components and features, we seek to provide clarity to developers and vendors of HIT products to facilitate product development, interoperability, and integration and data exchange with existing EMR/EHR products.
\end{abstract}

Keywords: Personal health records; Health informatics; Standards

\section{Introduction}

The health care system in the United States is one of the most expensive in the world with approximately $17.3 \%$ of GDP in annual expenses going to health care spending and with costs expected to rise dramatically by the end of the decade to nearly $20 \%$ of U.S. GDP [1]. In 2009 alone, nearly $\$ 2.5$ trillion was spent in the United States by federal, state, and local governments, corporations, and individuals on health care. Of this, it is estimated that nearly $\$ 700$ billion a year is spent on wasteful healthcare spending due to unnecessary care delivered, medical fraud, administrative inefficiency, provider error and medical mistakes, preventable conditions, and lack of care coordination [2]. To address the rising costs in health care, the Healthcare Information and Management Systems Society (HIMSS) submitted a series of recommendations for transforming the health care system through the use of health information technologies (HIT) [3]. In fact, HIT is a key component of the Health Care Reform Act adopted by Congress in March 2010. The term HIT is used to encompass numerous forms of digital health records such as electronic medical records (EMRs), electronic health records (EHRs), personal health records (PHRs), and payer-based health records (PBRs), each ideally integrating with one another seamlessly through common data information standards to promote interoperability, privacy and security of health information, and improve overall outcomes.

Hospital and insurer-based HIT adoption has dramatically increased over the past few years as a result of the Health Care Reform Act and has been a growing trend of the past decade. However, personal health records remain a challenge and could benefit greatly from a common set of standards. In its basic definition, PHRs provide a wide variety of tools allowing individuals to manage their health information in a variety of ways. Consumer driven, PHRs vary from traditional paper files kept by individuals of doctor's visits to more complex documentation covering the entire cradle-to-grave experience of each person. As a result of the heterogeneity of utilization by individuals, there is currently no common set of standards to define essential components of a PHR. Some argue for the bare minimum data content solely for emergency situations whereas others advocate for an entire lifetime health record. The American Health Information Management Association (AHIMA) defines a personal health record as "an electronic, lifelong resource of health information needed to make health decisions. Individuals own and manage the information in the PHR... from healthcare providers and the individual. The PHR is maintained in a secure and private environment, with the individual determining rights of access. The PHR does not replace the legal record of any provider"[4]. According to the definition by AHIMA and others, an individual ideally will be able to manage their personal health information (e.g. immunization records, allergies, medical test, etc.) from their own PHR and be able to exchange data with a wide variety of health care organizations and providers (i.e. primary care physician, pharmacies, hospitals, etc.).

Although, many organizations are attempting to develop a clear unique definition for PHRs, a real consensus has not been reached. Current standards do not take into consideration the uniqueness of the needs of the PHRs in contrast to other well-known technologies such as electronic hospital and payer-based health/medical records. While the focus of existing standards to date has been mainly on content of PHRs [5], consumer adoption and utilization of PHRs will be hindered

*Corresponding author: Binh Q. Tran, Department of Biomedical Engineering, The Catholic University of America, 620 Michigan Ave, NE Washington, DC 20064, Tel: (202)319-5181; E-mail: tran@cua.edu

Received November 28, 2011; Accepted January 21, 2012; Published January 26, 2012

Citation: Tran BQ, Gonzales P (2012) Standards and Guidelines for Personal Health Records in the United States: Finding Consensus in a Rapidly Evolving and Divided Environment. J Health Med Informat S6:001. doi:10.4172/2157-7420. S6-001

Copyright: $\odot 2012$ Tran BQ, et al. This is an open-access article distributed under the terms of the Creative Commons Attribution License, which permits unrestricted use, distribution, and reproduction in any medium, provided the original author and source are credited. 
until standards also include key features such as health management tools [6], medical scheduling [7], patient-provider communication [8], and medication/prescription management [9]. Seamless integration of data from provider sources is necessary to facilitate populating of the PHR and reduce the barrier to maintenance of the record. Further, the greatest value of PHRs will not just come from the data it stores, but from the various services it provides in order to provide individualized coordination of care needs. The objective of this research is to establish a recommended, minimum core for PHR data content (i.e. health information components) as well as key features (i.e. services) essential for widespread adoption by consumers and providers. This work provides a comprehensive review of existing standards and also a survey of current market vendors. A true PHR standard that addresses both data components as well as features will enable interoperability amongst PHRs as well as permit data to flow from electronic hospital and payer-based health/medical records and avoid "creating silos of data is just as wasteful as creating silos of care" [10].

\section{Review of existing PHR standards for health data and desired features}

Currently, there is a wide dispute on what health information should be contained in PHRs due to the complexity of the intended use by consumers. In addition to existing standards on data content, different professional organizations have made recommendations relating to PHR data content. In addition to AHIMA and HIMSS previously mentioned, other organizations who have developed content guidelines for PHRs include the National Academy of Science's Institute of Medicine (IOM), U.S. Department of Health \& Human Services' Agency for Healthcare Research and Quality (AHRQ), American Medical Informatics Association (AMIA), American Academy of Family Physicians (AAFP), and the National Medical Library Association/National Library of Medicine's Joint Electronic Personal Health Record Taskforce. This review provides a snapshot of an evolving landscape for PHRs.

\section{PHR standards}

Currently, there are numerous standards available that govern data content of PHRs. These include the Continuity of Care Document (CCD), ASTM's Continuity of Care Record (CCR), the HL-7 Clinical Document Architecture (CDA), Digital Imaging and Communications in Medicine (DICOM), and the Good Electronic Health Record (GEHR). The CCR contains "a core data set of the most relevant administrative, demographic, and clinical information facts about a patient's health care, covering one or more health care encounters"[11]. This standard is favored by AAFP [12]. In contrast, the HL-7's CDA was developed "to provide a common representation for clinical documents through a document mark-up standard" [13]. Whereas the former focuses on the patient's health care encounters through 17 different health categories, the latter focuses on documentation of clinical data such as consultation notes, pathology, radiology, discharge instructions, etc. In 2007, through a combined collaboration, the CCD, an XML-based standard with 16 health categories, resulted from the merging of two most popular standards for PHRs, the CCR and the CDA, and combines the benefits of both. "The Continuity of Care Document (CCD), resulting from the representation and mapping of CCR data within the CDA, will help drive the use of structured XML standards for clinical information exchange and the improvement of patient safety, quality, and efficiency"[14]. With its release, it was expected that the CCD would serve as the dominant standard for development of PHRs. However, in practice, this vision has yet to be proven realized.

In addition to the existing standards mentioned, individual professional organizations have proposed guidelines for PHR data content. AHIMA proposed a PHR with 18 health data categories intended as "a collection of important information about [one's] health or the health of someone you're caring for, such as a parent or a child that [one] actively maintains and updates" [15]. HIMSS provides an expanded set of guidelines beyond storage of health information to include support for "a collection of health related tools... for managing relevant health information, promoting health maintenance and assisting with chronic disease management" [16]. Similarly, in 2005, the AMIA proposed guidelines for PHRs to include tools allowing individuals to manage their health information, improve health and wellness, and contain both qualitative (i.e. description of symptoms or medical problems) and quantitative (i.e. blood pressure information, home diagnostic equipment, etc.) [17].

A comparison of the existing standards and guidelines is provided in table 1. From this data, 31 total data components were identified, twenty (20) common data components and 11 additional "other" components identified only once by any standard or guideline. Total data components for these standards and guidelines ranged from 9 (AMIA) to 18 (AHIMA). Only 5 data components (i.e. immunizations, medications/prescriptions, allergies/adverse reactions, family history, lab results) were identified by each of the seven standards/guidelines reviewed. One additional data component, procedures/surgeries, was identified by 6 of the 7. Five data components (i.e. health providers, insurance payer, social history/lifestyle, diagnoses/conditions, clinical encounters) were specified at least 4 times and 3 others (i.e. vital signs, care plan, home monitoring) were identified 3 times. Seven of the 11 "other" components, those identified only once, were contained in the PHR guideline set forth by AHIMA.

From table 1, we propose that a consensus set of data components can be derived. These are represented in table 2 and fall under three categories. Critical data components are comprised of those from table 1 that are identified by at least 6 of the 7 standards. Desired data components are those that were identified by 3-5 of the standards/ guidelines. Recommended data components were those mentioned by two of the standards/guidelines reviewed.

In addition to data standards, standards for terminology and nomenclature also have been adopted by PHR vendors. These include the International Classification of Diseases (ICD-9/ICD-10), the Systemized Nomenclature of Medicine (SNOMED) and the Unified Medical Language System (UMLS).These standards were developed to promote a common terminology to facilitate interoperability and enhance usability of PHR systems.

\section{Recommended PHR features}

While existing standards address data content and terminology, they do not include essential features (i.e. tools or services) that may make PHRs truly beneficial to consumers. Without these features, the impact of PHRs is likely to be muted and consumer adoption rates low. Through a review of the literature of existing pilot studies and surveys conducted, several key recommended features have emerged.

In 2002, a survey conducted by Capmed, one of the oldest PHR vendors, examined the primary use of PHRs by end-users. Results 
Citation: Tran BQ, Gonzales P (2012) Standards and Guidelines for Personal Health Records in the United States: Finding Consensus in a Rapidly Evolving and Divided Environment. J Health Med Informat S6:001. doi:10.4172/2157-7420.S6-001

Page 3 of 6

\begin{tabular}{|c|c|c|c|c|c|c|c|}
\hline & CCR & CCD & CDA & AHIMA & HIMSS & AMIA & ePHR Taskforce \\
\hline \multicolumn{8}{|l|}{ Common Data Components } \\
\hline Immunizations & $\mathrm{x}$ & $\mathrm{x}$ & $\mathbf{x}$ & $\mathrm{x}$ & $\mathbf{x}$ & $\mathbf{x}$ & $\mathbf{x}$ \\
\hline Medications/ Prescriptions & $\mathbf{x}$ & $\mathbf{x}$ & $\mathbf{x}$ & $\mathbf{x}$ & $\mathbf{x}$ & $\mathbf{x}$ & $\mathrm{x}$ \\
\hline Allergies/ Adverse Reactions & $\mathbf{x}$ & $\mathbf{x}$ & $x$ & $x$ & $\mathbf{x}$ & $x$ & $x$ \\
\hline Family History & $\mathrm{x}$ & $\mathbf{x}$ & $\mathbf{x}$ & $\mathbf{x}$ & $\mathbf{x}$ & $\mathbf{x}$ & $\mathbf{x}$ \\
\hline Lab/Test Results & $\mathbf{x}$ & $\mathbf{x}$ & $\mathbf{x}$ & $\mathbf{x}$ & $\mathbf{x}$ & $\mathbf{x}$ & $\mathrm{x}$ \\
\hline Procedures/ Surgeries & $\mathbf{x}$ & $x$ & $x$ & $x$ & & $\mathbf{x}$ & $x$ \\
\hline Health Providers & $x$ & & & & $\mathbf{x}$ & $\mathbf{x}$ & $\mathbf{x}$ \\
\hline Insurance / Payer Information & $\mathbf{x}$ & $\mathbf{x}$ & & $\mathbf{x}$ & $\mathbf{x}$ & & \\
\hline Social History/ Lifestyle & $\mathbf{x}$ & $\mathbf{x}$ & $\mathbf{x}$ & & & $\mathbf{x}$ & \\
\hline $\begin{array}{l}\text { Problems, Diagnoses, } \\
\text { Conditions }\end{array}$ & $x$ & $x$ & $x$ & & & & $x$ \\
\hline Clinical Encounter & $\mathbf{x}$ & $\mathbf{x}$ & $\mathbf{x}$ & & & & $\mathrm{x}$ \\
\hline Vital Signs & $\mathbf{x}$ & $\mathrm{x}$ & $\mathbf{x}$ & & & & \\
\hline Care Plan & $\mathbf{x}$ & $\mathrm{x}$ & $x$ & & & & \\
\hline Home Monitoring & & & $\mathbf{x}$ & & & $\mathbf{x}$ & $\mathbf{x}$ \\
\hline Medical Equipment & $x$ & $\mathbf{x}$ & & & & & \\
\hline Functional Status & & $x$ & $x$ & & & & \\
\hline Health Maintenance/ Exercise & & & & $\mathbf{x}$ & $\mathbf{x}$ & & \\
\hline Advanced Directives & & $\mathrm{x}$ & & $\mathrm{x}$ & & & \\
\hline Personal ID (i.e. SSN, etc) & & & & $\mathbf{x}$ & $\mathbf{x}$ & & \\
\hline Emergency Contact Info & & & & $\mathbf{x}$ & $\mathbf{x}$ & & \\
\hline \multicolumn{8}{|l|}{ Other Items: } \\
\hline & $\begin{array}{l}\text { Patient Demographics } \\
\text { - Statistics } \\
\text { - Appropriate Results }\end{array}$ & - Purpose & & 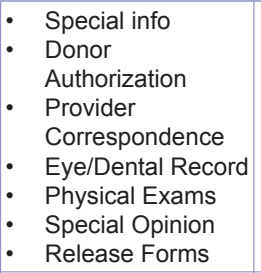 & & & \\
\hline Total Data Components & 17 & 16 & 13 & 18 & 10 & 9 & 10 \\
\hline
\end{tabular}

Note: Grey: Indicates data components identified in at least 6 of the 7 PHR standards. Green: Indicates components identified by $3-5$ of the 7 PHR standards. White: Indicates data components identified in 2 or less of the 7 PHR standards.

Table 1: Comparison of data components for existing standards and guidelines.

showed $26.4 \%$ would like to access family medical history, $21.4 \%$ track doctor visits, $18.7 \%$ track lab results, and $13.5 \%$ track/trend one's health status [18]. In 2003, the Markle Foundation conducted a national survey of 1,246 online households who indicated $75 \%$ will use a PHR to email their doctor, $69 \%$ to track their immunizations, $69 \%$ to note mistakes in their record, $65 \%$ to transfer information to a new doctor, $63 \%$ to get and track their test results [19]. Additional recommendations by the Institute of Medicine (IOM) proposed PHR access to a provider's system for data exchange and PHR-facilitated communication between physician and individuals [20]. Additionally, the AMIA, Kaiser Permanente Institute for Health Policy, the Robert Wood Johnson Foundation, and HHS' AHRQ proposed features for facilitating gathering of data from different health care sources [21]

\begin{tabular}{|c|c|c|}
\hline Critical & Desired & Recommended \\
\hline Immunizations & Health Providers & Vital Signs \\
\hline Medications/ Prescriptions & Insurance / Payer Information & Care Plan \\
\hline $\begin{array}{c}\text { Allergies/ Adverse } \\
\text { Reactions }\end{array}$ & Social History/ Lifestyle & Home Monitoring \\
\hline Family History & $\begin{array}{c}\text { Problems, Diagnoses, } \\
\text { Conditions }\end{array}$ & \\
\hline Lab/Test Results & Clinical Encounter & \\
\hline Procedures/ Surgeries & & \\
\hline
\end{tabular}

Table 2: Recommended Consensus Standard for PHR Data Components.

\begin{tabular}{|l|c|c|}
\hline \multicolumn{2}{|c|}{} & Free \\
\hline Categories & 25 & 63 \\
\hline Web-Based & 0 & 1 \\
\hline Software Based & 2 & 13 \\
\hline
\end{tabular}

Table 3: PHR Vendors by Platform and Cost.

(i.e. claims data, commercial laboratories, monitoring devices, etc) and for providing ongoing and secure EMAIL communication between physician and individual, online appointment scheduling, collaborative health tracking and chronic disease management [17], prescription refills, medication reminders, and to report problems. [20]

\section{Review of current PHR vendors and products}

As shown in table 1, the varied data components specified in each of the existing standards and guidelines led to a diverse offering of PHR products. A report on standard adoption made by Joint Electronic Personal Health Record Task Force indicated a lack of standard adoption among PHR vendors [22]. The reports highlights from a total of 117 PHR vendors sampled, only $34 \%$ (44) followed some kind of standard. This suggests that the remainder may not follow any standard. Also, the breakdown of standard adoption varies based on the type of standard they follow: 16 PHRs vendors followed the CCR, 11 HL7, and 5 CCD. 
Citation: Tran BQ, Gonzales P (2012) Standards and Guidelines for Personal Health Records in the United States: Finding Consensus in a Rapidly Evolving and Divided Environment. J Health Med Informat S6:001. doi:10.4172/2157-7420.S6-001

Page 4 of 6

\begin{tabular}{|c|c|c|c|}
\hline About My Health & Health String & Medi Connect Global & Patient Power \\
\hline Access My Records & Health Records Online & MedicalSummary & PatienTrak \\
\hline Activ Doctors Online & HealthTracer & MedNotice & People Chart \\
\hline AMESMyFile & HealthTracks & Medsfile.com & Personal MD \\
\hline A Smart PHR & HealthTrio & MemiTech 911 Medical ID Card & Personal Medical Records \\
\hline CapMedPHR & IHealthRecord & Merck Source & PHR4me \\
\hline CapMed icePHR Mobile & IQHealth & Microsoft Healthvault & Polka \\
\hline Caregiver Alliance Web Services & It runs in my family & MyChart & Powerful Patient Solutions \\
\hline Compiling Your Family Health History & Jakoter Health Organizer & My Doclopedia PHR & RelayHealth \\
\hline CrisisID & Juniper Health & My Health & RememberltNow \\
\hline dLife & Keas & MyHealthFolders & Securamed \\
\hline DrGlobe.com & KIS Electronic Health Record & My HealtheVet & So Tell Me Medical Organizer \\
\hline Dr. I-net & Laxor & MylifeSaver & Synchart \\
\hline Dossia & Lifeledger & MyMedSafe & TAC Health \\
\hline EMRy Stick & LifeNet & MyMedicalRecords.com & Telemedical.com \\
\hline ER-IDcard & LifeonKey & MyMediConnect & VIA \\
\hline Full Circle Registry & LifeSensor & MyMediList & VitalChart \\
\hline GlobalPatientRecord & Lynxcare & MyNetRecord.com & Web MD Health Manager \\
\hline Good Health Network & Magnus Health Portal & My Personal Guardian & WorldMedCard \\
\hline GoogleHealth & MdiKeepr & myPHI & Your Health Record \\
\hline HealthButler & MedDataNet & MyPRO Medical-Health Records Organizer & ZebraHealth \\
\hline Healthy Circles & Medefile & My Vital Data & 911 Medical ID Card \\
\hline HealtheTracks & Medical ID Card & NoMoreClipboard & \\
\hline Healthgram.com & MedicAlert & Organized Solutions & \\
\hline HealthNote & MediCompass & OnlineMedicalRegistry & \\
\hline
\end{tabular}

Table 4: Sample list of commercially available PHR products.

\begin{tabular}{|c|c|c|c|c|c|c|c|c|c|c|c|}
\hline Item \# & $\begin{array}{c}\text { Data } \\
\text { Components }\end{array}$ & 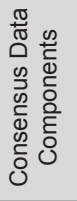 & 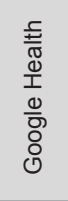 & 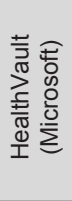 & 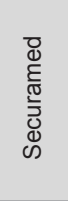 & 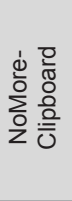 & 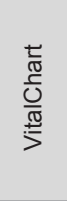 & 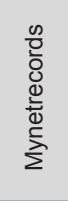 & $\frac{\sum_{0}^{0}}{30}$ & $\begin{array}{l}\bar{\pi} \\
\frac{0}{0} \\
0 \\
\dot{0}\end{array}$ & 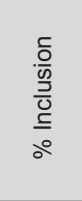 \\
\hline 1 & Immunization & C & $\mathbf{x}$ & $\mathbf{x}$ & $\mathbf{x}$ & $\mathbf{x}$ & & $x$ & $x$ & & $75 \%$ \\
\hline 2 & Medications/Prescriptions & C & $x$ & & & $\mathbf{x}$ & $x$ & $\mathbf{x}$ & $x$ & $x$ & $75 \%$ \\
\hline 3 & Allergy/Adverse Reactions & $\mathrm{C}$ & $x$ & $x$ & $x$ & $x$ & $x$ & $\mathbf{x}$ & $x$ & $x$ & $100 \%$ \\
\hline 4 & Family History & $\mathrm{C}$ & & $\mathbf{x}$ & & $\mathbf{x}$ & $\mathbf{x}$ & $\mathbf{x}$ & & $x$ & $62.5 \%$ \\
\hline 5 & Lab/Test Results & C & $\mathbf{x}$ & & $x$ & & $x$ & & $x$ & & $50 \%$ \\
\hline \multirow[t]{2}{*}{6} & Procedures/Surgeries & C & $\mathbf{x}$ & $\mathbf{x}$ & & $\mathbf{x}$ & $\mathbf{x}$ & & $\mathbf{x}$ & & $62.5 \%$ \\
\hline & $\%$ of Critical Data Components & $\mathrm{N}=6$ & $83 \%$ & $66 \%$ & $50 \%$ & $83 \%$ & $83 \%$ & $66 \%$ & $83 \%$ & $50 \%$ & \\
\hline 7 & Health Providers & $\mathrm{D}$ & & & & & & $x$ & & & $12.5 \%$ \\
\hline 8 & Insurance/Payer Information & $\mathrm{D}$ & $\mathbf{x}$ & $x$ & $x$ & $x$ & $x$ & & $x$ & $x$ & $87.5 \%$ \\
\hline 9 & Social History/Lifestyles & $\mathrm{D}$ & & & $x$ & $\mathbf{x}$ & $\mathbf{x}$ & $x$ & & & $50 \%$ \\
\hline 10 & Problems/Diagnoses/ Conditions & $\mathrm{D}$ & $\mathbf{x}$ & $\mathbf{x}$ & $x$ & & $x$ & & $x$ & & $62.5 \%$ \\
\hline \multirow[t]{2}{*}{11} & Clinical Encounters & $\mathrm{D}$ & & & & & & & & & $0 \%$ \\
\hline & $\%$ of Desired Data Components & $\mathrm{N}=5$ & $40 \%$ & $40 \%$ & $60 \%$ & $40 \%$ & $60 \%$ & $40 \%$ & $40 \%$ & $20 \%$ & \\
\hline 12 & Vital signs & $\mathrm{R}$ & & & & & & & & & $0 \%$ \\
\hline 13 & Care Plan & $\mathrm{R}$ & & & & & & & & & $0 \%$ \\
\hline \multirow[t]{2}{*}{14} & Home Monitoring & $\mathrm{R}$ & & & & & & & & & $0 \%$ \\
\hline & $\begin{array}{l}\% \text { of Recommended Data } \\
\text { Components }\end{array}$ & $\mathrm{N}=3$ & $0 \%$ & $0 \%$ & $0 \%$ & $0 \%$ & $0 \%$ & $0 \%$ & $0 \%$ & $0 \%$ & \\
\hline
\end{tabular}

Note: For data components, $\mathrm{C}=$ critical; $\mathrm{D}=$ desired; $\mathrm{R}=$ recommended.

Table 5: Comparison of Ideal Data Components vs. PHR Products. 


\begin{tabular}{|c|c|c|c|c|c|c|c|c|c|}
\hline $\begin{array}{l}\text { Desired } \\
\text { Features }\end{array}$ & 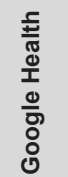 & 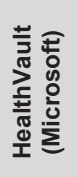 & 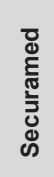 & 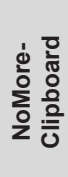 & 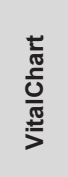 & 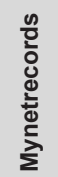 & $\frac{\sum_{0}^{0}}{30}$ & $\begin{array}{l}\bar{\pi} \\
\frac{0}{0} \\
\text { 음 } \\
\dot{\Delta}\end{array}$ & $\frac{\text { 음 }}{\frac{c}{0}}$ \\
\hline Access to outside data (i.e. EHRs, providers) & $\mathbf{x}$ & $\mathbf{x}$ & & $\mathbf{x}$ & & & & & $37.5 \%$ \\
\hline Interaction $\mathrm{w} /$ physician & & & & & $\mathbf{x}$ & & & & $12.5 \%$ \\
\hline Access to reliable health information & & & & & & & & & $0 \%$ \\
\hline Health Tracking & $\mathbf{x}$ & $\mathbf{x}$ & & & & & $\mathbf{x}$ & $\mathbf{x}$ & $50 \%$ \\
\hline$\%$ of Desired Features & $50 \%$ & $50 \%$ & $0 \%$ & $25 \%$ & $25 \%$ & $0 \%$ & $25 \%$ & $25 \%$ & \\
\hline
\end{tabular}

Table 6: Comparison of Proposed Features vs. PHR Products.

Additionally, the availability of PHR products vary by platform from paper-based to software-based (i.e. applications) to web-based and also be separated by price, i.e. free vs. charge. Using the AHIMA operated website, myPHR.com, a search of all platforms (i.e. paper, software, and web) and price categories resulted in 97 unique PHR products available. Table 3 provides a summary of commercially available products by platform and price categories. Further, table 4 provides a listing of the various available products currently on the market. As the PHR marketplace is a rapidly changing and fluid marketplace, this information only provides a current snapshot of vendors and products available. It is expected this list will be significantly different within a few years as vendors seek to develop a business model around PHRs. Already within the past 6 months, Google has announced its exit from the PHR market.

To assess the level of agreement of existing PHR products to the proposed consensus standards listed in table 2, a sample of $8 \mathrm{PHR}$ products from table 4 were evaluated. This sample was selected based upon their importance to the PHR market. These include PHR offerings such as Google Health (Google Inc., Mountain View, CA), Healthvault (Microsoft Corp., Redmond, WA), NoMoreClipboard (Ft. Wayne, IN) , VitalChart (Durham, NC), MyNetRecords (Leichhardt, Australia), WebMD (NewYork, NY), Dr. Global (DGL, New Plymouth, New Zealand), Securamed (http://www.securamed.com). Table 5 provides a comparison of how each product compares to the proposed consensus data standard.

Of the PHR products evaluated, none contained all critical components proposed. The percent inclusion, i.e. percentage of times a particular data component was found in all PHRs surveyed, ranged from $50 \%$ to $100 \%$. Surprisingly, the only critical data component to be included in $100 \%$ of PHRs surveyed was "allergies/adverse reactions." For each PHR surveyed, the percentage of critical data components ranged from $50 \%$ to $83 \%$. Half of the PHRs evaluated contained 5 of the 6 critical components. Of the desired data components proposed in table 2, the level of inclusion is drastically lower. Again, none of the desired data components was contained in all PHRs tested. One data component, insurance/payer information, was found in 7 of the 8 PHRs evaluated. One category, clinical encounters, was not found in any of the PHRs, while another, health providers, was found in only one. Finally, evaluation of the recommended data components shows that none of the PHR vendors included these any of these data components.

Finally, table 6 provides a comparison of the same PHR products in relation to the desired features (i.e. services) mentioned previously. Overall, there is a low level of inclusion of these features across the various PHR products surveyed. The same can be observed for each individual PHR with only Google Health and Microsoft's Health vault containing at least $50 \%$ of the desired features.

\section{Conclusion}

The findings of this research suggest that, while there is a strong desire to establish standards for data components contained in PHRs, there remain considerable differences amongst the various existing standards and guidelines for PHRs. We propose herein a consensus standard for PHR data components consisting of 14 total data components. These 14 data components fall under three main categories, i.e. critical (i.e. immunizations, medications, allergies, family history, lab/test results, procedures/surgeries), desired (i.e. health providers, insurance/payer information, lifestyle/social history, conditions and diagnoses, clinical encounters), and recommended (i.e. vital signs, care planning, home monitoring). Of these, we place the highest priority upon the components contained in the critical and desired categories and believe these are 11 data components essential for all PHRs.

From a sample of 8 PHR vendors selected based upon size and influence within the market, we observed there exists a low level of adoption of existing PHR standards, consistent with previous reports in the literature [22]. For critical data components, inclusion into existing PHR products range greatly by vendor between $50-83 \%$. For desired data components, inclusion by product ranged between $40-60 \%$. None of the PHR products tested included any of the data components from the recommended category. The results from the review of PHR vendors perhaps are reflective of the confusion in the marketplace regarding which standard or guideline is most appropriate to adopt. By this effort, we have attempted to provide some clarity for PHR developers and vendors.

In addition to proposing a consensus standard for PHR data components, this research propose a minimum set of features would also enhance adoption of PHRs amongst consumers. These key features include services to 1) link other existing sources of medical information (i.e. EHRs, providers, pharmacies, insurance companies) to the PHR under the control of the individual, 2) enhance interaction with health care providers, and 3) access to reliable health information, and 4) health tracking and monitoring. In order for consumers to be actively engaged in their own health and care, the PHR must serve not only to record an individual's health throughout the lifespan, but must also facilitate healthy living, chronic disease management, and preventive models of health care. 
Citation: Tran BQ, Gonzales P (2012) Standards and Guidelines for Personal Health Records in the United States: Finding Consensus in a Rapidly Evolving and Divided Environment. J Health Med Informat S6:001. doi:10.4172/2157-7420.S6-001

\section{References}

1. Jones B (2010) Medical expenses have very steep rate of growth. USA Today

2. Gellner R (2009) $\$ 700$ billion waste in annual waste within health care system would pay for reform.

3. HIMSS.( 2009) Enabling Health Reform Using Information Technology.

4. AHIMA e-HIM Personal Health Record Working Group (2005) The Role of the Personal Health Record in the EHR. J of AHIMA 76: 64 A-64D.

5. Adams J , Bakalar R, Kaufman J, Mounib E, (2008) Healthcare 2015 and Personal Health Records: A Standards Framework. IBM Institute for Business Value, Somers, NY.

6. Tang PC, Ash JS, Bates DW, Overhage JM, Sands DZ (2005) Personal Health Records: Definitions, Benefits, and Strategies for Overcoming Barriers to Adoption. J Am Medical Informatics Assoc 13: 121-126.

7. U.S. Dept Health and Human Services Center for Disease Control and Prevention, Personal Health Records and Personal Health Record Systems. A Report and Recommendations from the National Committee on Vital and Health Statistics: 1-35, 2006.

8. Cimino JJ, Patel VL, Kushniruk AW (2002) The Patient Clinical Information System (PatCIS): Technical solutions for and experience with giving patients access to their electronic medical records. Int J of Medical Informatics 68: 113127

9. Cognetti G, Cecere L (2003) E-oncology and health portals: Instructions and standards for the evaluation, production organization and use. J of Exp \& Clinical Cancer Res 22: 677-686.

10. Tang PC and Lansky D (2005) The Missing Link: Bridging the Patient-Provider Health Information Gap. Health Affairs 24: 1290-1295.

11. CorePoint Health, Understanding the Continuity of Care Record, 2009

12. Endsley S, Kibbe DC, Linares A, Colorafi K (2006) An Introduction to Personal
Health Records. Fam Pract Manag.

13. Ferranti JM, Musser RC, Kawamoto K, Hammond WE (2006) The Clinical Document Architecture and the Continuity of Care Record: A Critical Analysis. J Am Med Inform Asso 13: 245-252.

14. Shindler, B, HL7 Continuity of Care Document, a Healthcare IT Interoperability Standard, is Approved by Balloting Process and Endorsed by Healthcare IT Standards Panel. ASTM: 1-3, 2007.

15. Ball MJ and Gold J (2006) Banking on Health: Personal Health Records and Information Exchange. J. of Healthcare Info Management 20: 71-83.

16. HIMSS Personal Health Records (PHR) Steering Committee, Personal Health Records: A HIMSS Definition and Position Statement2007.

17. American Medical Informatics Assoc, The Transformative Potential of Integrated Personal Health Records [Discussion Draft], 2006. http://www2. amia.org/gotehr/docs/phrwhitepaperdraftvfinal.pdf

18. Cronin C (2006) Personal Health Records: An Overview of What Is Available to the Public. AARP.

19. Markle Foundation (2003) Connecting for Health, A Private Public Collaborate. The Personal Health Working Group: 1-58.

20. Ball MJ, Smith C, and Bakalar R, Personal Health Records: Empowering Consumers. J of Healthcare Info Management 21:1

21. Detmer D, Bloomrosen M, Raymond B, Tang P (2008) Integrated Persona Health Records: Transformative tools for consumer centric care. BMC Medical Informatics and Decision Making: 1-14.

22. Jones DA, Shipman JP, Plaut DA, Selden CR (2010) Characteristics of personal health records: Findings of the Medical Library Association/National Library of Medicine Joint Electronic Personal Health Record Taskforce, J Med Libr Assoc 98: 243-249. 